\title{
The COSPAR Capacity-Building Workshop Programme, 2000-2007
}

\author{
Keith A. Arnaud ${ }^{1,2}$ and A. Peter Willmore ${ }^{3} \dagger$ \\ ${ }^{1}$ Laboratory for X-ray Astrophysics, NASA GSFC, Greenbelt, MD 20771, USA \\ ${ }^{2}$ Department of Astronomy, University of Maryland, College Park, MD 20742 USA \\ email: kaa@milkyway.gsfc.nasa.gov \\ ${ }^{3}$ School of Physics and Astronomy, University of Birmingham, Birmingham, B15 2TT, UK \\ email: apw@star.sr.bham.ac.uk
}

\begin{abstract}
The Committee on Space Research (COSPAR) Capacity-Building Programme introduces astronomers in the developing world to the rich resource of space research online archives. The programme consists of a series of regional workshops which each bring together about 30 developing world astronomers with around 8 teaching faculty for lectures and hands-on projects. Five workshops have been held so far with another two planned for next year and a budget which enables on average one workshop each year. Proposals for future workshops are encouraged.
\end{abstract}

Keywords. methods: data analysis

\section{Introduction}

Space missions being rare, expensive, and generally short-lived have long had a policy of archiving all their observational data to ensure that these resources are not lost. In the past these data were only available to researchers at the institutions running the space missions. However the rise of the Internet and World Wide Web has changed this. Extensive online archives and the software to use them are now available for all major space research missions, with smaller missions being served by archival research centers. In addition many missions are open world-wide to observing proposals and archival research can provide a stepping stone towards collaborations or even acquiring one's own dedicated observations.

Most users of these archives are from the US, Europe, and Japan with relatively few from the developing world. There are a number of likely reasons for this: the poor quality of internet connections in much of the developing world; a lack of awareness that such archives are freely available, along with a belief that space data are for people who build space missions; research programmes in developing countries are built around affordable ground-based facilities; cultural factors such as conservatism among graduate supervisors.

To help more astronomers in the developing world make use of space research archives COSPAR has organized a series of practical workshops centred on current, active space missions. The aims are to ensure that all participants leave with the ability to set up and use software and data at their own institutions without further support. The workshops also serve to build links between participants and lecturers, as well as among the participants.

$\dagger$ Paper presented by K.A.; A.P.W was the original invited speaker 
Table 1. COSPAR workshops and local projects in italics.

\begin{tabular}{llll}
\hline 2001 & X-ray astronomy & Chandra, XMM-Newton & INPE, Brazil \\
2003 & X-ray astronomy & Chandra, XMM-Newton, Astrosat & Udaipur, India \\
2004 & Magnetospheric physics & Cluster, Double-Star & Beijing, China \\
2004 & X-ray astronomy & Chandra, XMM-Newton, SALT & Durban, South Africa \\
2005 & Space Oceanography & GOOS & CRTS, Rabat, Morocco \\
\hline
\end{tabular}

\section{The history of the programme}

The COSPAR programme of capacity-building workshops was established in 1999 . The first two workshops concentrated on X-ray astronomy because this was the field of the instigator of the programme (APW) and with two major observatories, Chandra from NASA and XMM-Newton from ESA, the field is particularly dynamic at present. Following the initial success a panel to manage the programme was set up in 2004 with an annual budget large enough to fund approximately one workshop per year. Table 1 lists the workshops held. The India, China, and South Africa workshops also included discussion of a local project with applicability to the workshop theme. These projects are shown in italics in Table 1.

The workshops are held in a developing country university or space centre with good facilities and access to the internet. Participants are then drawn from the host country and its neighbours. For instance, the workshop in South Africa catered to participants from a range of sub-Saharan countries. There are usually 25-30 participants chosen by the organizing committee from applicants and about 8 lecturers, both local and foreign experts. The participants are generally senior postgraduate students, post-docs, or young faculty. All their expenses are covered by COSPAR and the co-sponsors.

COSPAR covers $50-60 \%$ of the cost, the host country $15-20 \%$ (usually US $\$ 10,000$ $15,000)$ and the rest from a range of partners, one of the most important being the IAU. Other past partners have been ICSU, UNESCO/IOC, UN/OOSA, URSI, Abdus Salam ICTP, US National Academy of Science, ESA, NASA, IFREMER, IRD, ISPRS, Medias France, UK Met Office, Univ. of Plymouth, MERCATOR OCEAN MERSEA Project.

The host country organizations provide considerable logistical support in addition to contributing to the funding and the workshops would not be possible without their enthusiastic help. For past workshops these organizations have been FAPESP Brazil, Indian Space Research Organization, Physical Research Lab. Ahmedabad, CSSTEAP India, CCSAR China, South African National Research Foundation, and CRTS Morocco.

There are currently two more workshops approved: magnetospheric physics in Sinaia, Romania in June, 2007 organized by Joachim Vogt, Thierry Dudok de Wit, and Octav Marghitu; and planetary science in Montevideo, Uruguay also in 2007 organized by Gonzalo Tancredi and Mike A'Hearn. If funds allow, there may be another applications workshop in 2007; otherwise this may be in 2008. There is also an attempt underway to organize a Chandra/XMM-Newton workshop at the Library of Alexandria in Egypt, however this will not be fully funded by COSPAR and depends on obtaining sufficient funds from other sources.

We encourage proposals for workshops. Guidelines are available from the COSPAR website (www.cosparhq.org) or by contacting one of the authors (APW). 


\section{Workshop structure}

The available workshop time is split about equally between lectures and a data analysis project. The lectures aim to give the participants a basic understanding of the topic, including a description of the mission(s), the software, and the sorts of science that they make possible. More basic topics are sometimes necessary, for instance a series of lectures on statistics was included in the X-ray astronomy workshops.

The data analysis project is the key element of the workshop. Each participant has a dedicated PC for the duration of the workshop on which they work on data analysis under the direction of an advisor who is one of the lecturers. An attempt is made to match the project to the participant's research interests and to those of their home institution. Participants are also encouraged to form teams if they have similar interests.

The workshop closes with a poster session where each team presents a standard meeting poster on the work they have accomplished on their project. Some of these posters have been very impressive.

\section{Success of the workshops}

To judge the impact of the programme a questionnaire was e-mailed to the participants of the Brazil workshop two years after the event. Of the $50 \%$ who replied, all regarded the workshop as having been valuable for their careers, $86 \%$ had used Chandra or XMMNewton data or both, $70 \%$ were still in contact with one or more of the lecturers, $70 \%$ had a publication either in press or in preparation which was based at least in part on Chandra or XMM-Newton data. All the respondents regarded the project as a key element in the success of the workshop.

There is other more anecdotal evidence for the success of the programme. Guest observer proposals for the Chandra satellite from Latin America doubled in number the year after the Brazil workshop. Following the India workshop, two institutes in Bangalore established a joint research programme in X-ray astronomy. Finally, approximately 10 graduates of the workshops attended the 2006 Beijing assembly of COSPAR and presented papers.

\section{Conclusion: challenges and questions}

There have been a number of challenges to be met during these workshops. The working language is English and there have been communication difficulties in a few cases. It certainly helps to have lecturers fluent in all the languages spoken by the participants. Another problem has been participant familiarity with Linux, which is usually required to run the data analysis software. Participants from countries with less well-developed scientific infrastructure are more likely to be familiar with Windows than Linux and this provides a steep learning curve before they can start doing anything useful. With the adoption of Linux systems by a number of developing country governments this problem may not be as serious in future.

Another challenge is how to follow up these workshops. It may be difficult for a participant to apply what they have learnt when they return to their home institution. The hope has been that the workshops lead to regional networking but there has been no formal structure put in place to ensure this happens. Finally, it is worth considering how this programme fits in with other developing world initiatives, some of which are described in other papers in this volume. Should COSPAR continue going it alone with these workshops or look to fit them into some larger structure? 


\section{Acknowledgements}

This programme is now rolling and $\approx 135$ mostly young scientists have taken part as participants. It depends crucially on: those who have funded it; the local organizers Joao Braga and Tania Sausen (Brazil), H.S.S. Sinha (India), Ji Wu (China), Arthur Hughes (South Africa) and Driss el Hadani (Morocco), and, in the cases of the Beijing and Rabat workshops, the main directors Joachim Vogt and Thierry Dudok de Wit (Beijing) and Jean-Louis Fellous and Raymond Zaharia (Rabat); the lecturers who give up two full weeks of their time for an activity that brings no direct returns in research. We thank them all.

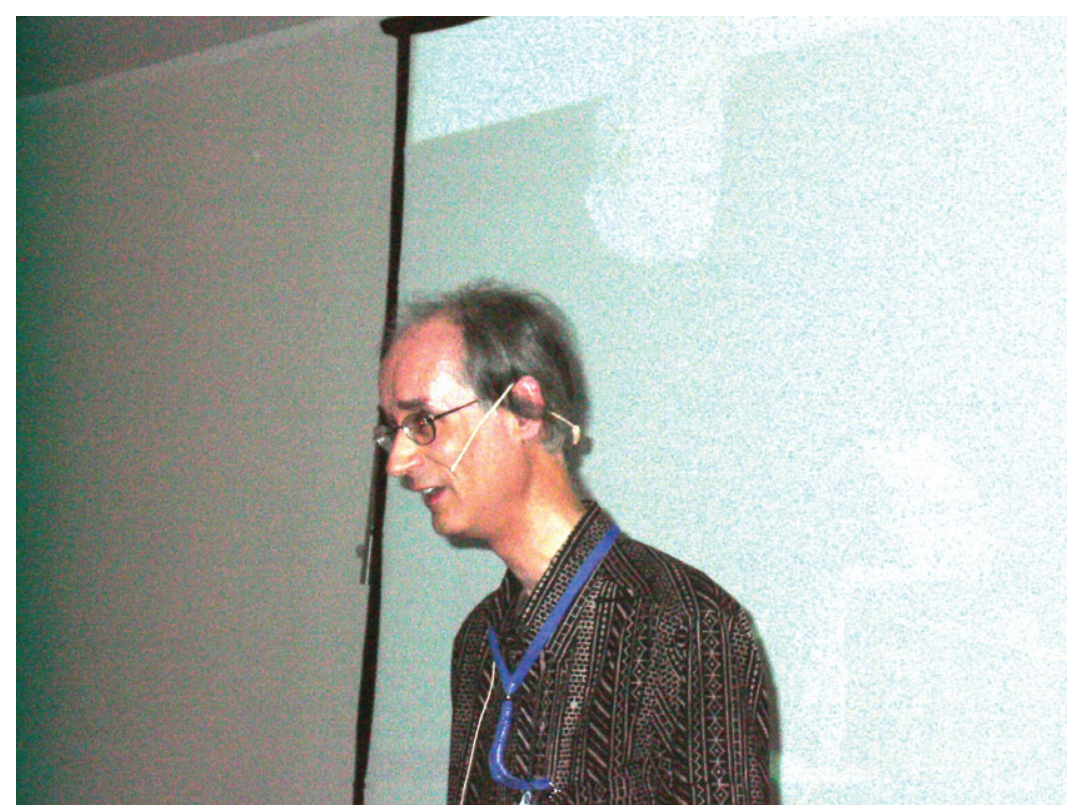

Keith Arnaud 